\title{
Parkinsonian Symptomatology May Correlate with CT Findings before and after Shunting in Idiopathic Normal Pressure Hydrocephalus
}

\author{
Mitsuaki Ishii, ${ }^{1,2}$ Toshio Kawamata, ${ }^{1}$ Ichiro Akiguchi, ${ }^{3}$ Hideo Yagi, ${ }^{3}$ Yuko Watanabe, ${ }^{3}$ \\ Toshiyuki Watanabe, ${ }^{3}$ and Hideaki Mashimo ${ }^{4}$ \\ ${ }^{1}$ Department of Rehabilitation Science, Kobe University Graduate School of Health Sciences, Kobe 654-0142, Japan \\ ${ }^{2}$ Department of Physical Therapy, Bukkyo University School of Health Science, Kyoto 603-8301, Japan \\ ${ }^{3}$ Center of Neurological and Cerebrovascular Disease, Koseikai Takeda Hospital, Kyoto 600-8558, Japan \\ ${ }^{4}$ Department of Physical Therapy, Maizuru Municipal Hospital, Kyoto 625-0035, Japan
}

Correspondence should be addressed to Toshio Kawamata, kawamata@kobe-u.ac.jp

Received 30 September 2009; Revised 28 December 2009; Accepted 23 January 2010

Academic Editor: Hélio Teive

Copyright (c) 2010 Mitsuaki Ishii et al. This is an open access article distributed under the Creative Commons Attribution License, which permits unrestricted use, distribution, and reproduction in any medium, provided the original work is properly cited.

\begin{abstract}
We aimed to investigate the characteristics of Parkinsonian features assessed by the unified Parkinson's disease rating scale (UPDRS) and determine their correlations with the computed tomography (CT) findings in patients with idiopathic normal pressure hydrocephalus (iNPH). The total score and the scores for arising from chair, gait, postural stability, and body hypokinesia in the motor examination section of UPDRS were significantly improved after shunt operations. Stepwise multiple regression analysis revealed that postural stability was the determinant of the gait domain score of the iNPH grading scale. The canonical correlation analysis between the CT findings and the shunt-responsive Parkinsonian features indicated that Evans index rather than midbrain diameters had a large influence on the postural stability. Thus, the pathophysiology of postural instability as a cardinal feature of gait disturbance may be associated with impaired frontal projections close to the frontal horns of the lateral ventricles in the iNPH patients.
\end{abstract}

\section{Introduction}

Symptoms of idiopathic normal pressure hydrocephalus (iNPH) are potentially reversible if diagnosed and treated in the early stage of the disease $[1,2]$, and these symptoms may be alleviated by shunt operations. Although iNPH is referred to as "treatable dementia," gait disturbance occurs earlier and is usually the most frequent symptom; improvement after shunt operation is more likely to occur in this symptom than the other symptoms [3]. Thus, it has been proposed that iNPH be also recognized as a "treatable gait disorder" [4]. Gait disturbance in iNPH is characterized by petitpas gait, magnetic gait, and broad-based gait [5]. It is believed that iNPH is one of the causes of higher-level gait disorders characterized by a combination of the features of postural instability, hypokinesia, and gait ignition failure [6].
It has also been reported that patients with iNPH exhibit characteristic radiographic abnormalities [7]. Computed tomography (CT) or magnetic resonance imaging (MRI) show enlarged ventricles, frequent white matter lesions [8], increased sylvian fissures space, decreased superior convexity, and medial subarachnoid space; further, in some patients, focally dilated sulci were observed over the convexity of the hemisphere [7]. More recently, decreased diameters of the midbrain have been reported [9]. A few studies report the correlation between gait disturbances and radiographic abnormalities in iNPH $[9,10]$. However, whether the severity of the Parkinsonian features can be assessed by the radiographically detected morphologic alterations remains to be determined.

In addition to characteristic gait disturbances, patients with iNPH exhibit parkinsonism [11-13]. Thus, the unified Parkinson's disease rating scale (UPDRS), which is 
widely used in the clinical assessment of Parkinson's disease [14], may be used for the evaluation of iNPH. However, investigations on the evaluation of the characteristics of the parkinsonism by using the UPDRS and the correlation between the rating of this scale and the radiographic abnormalities observed in iNPH have not been reported.

To define the characteristic signs and symptoms, an improvement in the symptoms should be observed following a shunt operation. However, previous studies on the correlation between movement disorders and radiographic abnormalities in iNPH have not considered the importance of the above feature.

Our aims were (1) to investigate the characteristics of Parkinsonian features, (2) to explore whether a relationship exists between the parkinsonism, especially those assessed by the UPDRS, and the morphologic changes observed radiographically; (3) and to investigate the correlation between the gait domain score of the iNPH grading scale (iNPHGS) [15] and the motor score of the UPDRS in patients diagnosed with definite iNPH.

\section{Methods}

2.1. Subjects. On the basis of clinical guidelines for iNPH, 13 participants (11 males and 2 females) were diagnosed with definite iNPH [4]. They were admitted to the neurological clinic at the Department of Neurology of Koseikai Takeda Hospital or the Department of Neurosurgery of Maizuru Municipal Hospital between August 2004 and January 2006. Their ages ranged from 66 to 81 years with a mean age of 77.0 years (standard deviation (SD) 4.3). All the subjects were informed about the details of the study, and they agreed to participate and provided written informed consent. All the procedures in this study were performed according to the clinical study guidelines of the local ethics committee and were approved by the internal review board.

The clinical guidelines for iNPH propose 3 levels of diagnosis: possible, probable, and definite iNPH. The diagnosis of "possible" iNPH is based on the presence of 1 or more classical symptoms (gait disturbance, cognitive impairment, and urinary incontinence), ventricular dilation (Evans index: maximal width of frontal horns/maximal width of inner skull $>0.31$ ) with closing sulci at high convexity, and clear cerebrospinal fluid (CSF) with a normal CSF pressure in middleaged and elderly patients. The diagnosis of "probable" iNPH is based on improved gait after the CSF tap test or continuous CSF drainage. The diagnosis of "definite" iNPH is based on the improvement of symptoms after a CSF shunt operation [4]. We excluded patients with NPH secondary to disorders such as subarachnoid hemorrhage.

The Evans index of all patients was more than 0.31 . The MRIs of all patients revealed increase in the width of the sylvian fissure and reduction in the width of the cortical sulcal space in the superior convexity [7]. All patients showed frontal release signs such as sucking, grasping, or palmomental reflex. The patients took no medications including levodopa or dopamine agonists before undergoing ventriculoperitoneal shunt (VP shunt) operations. We employed a Codman-Medos adjustable valve for the shunt system.

\section{Procedure and Data Analyses}

3.1. Assessment of Parkinsonism. The Parkinsonian features were assessed by a physical therapist, in terms of the subitems and the total scores of the motor examination section of the UPDRS and the iNPHGS. The best score after 3 measurements was used as the final score. The motor examination section of the UPDRS comprises 14 items. All the items are graded on a 5-point scale, from 0 to 4 [14]. The severity of gait disturbance was semiquantified according to the iNPHGS gait domain score [16]. The iNPHGS gait domain is graded as 0 (normal), 1 (dizziness or awareness of gait disturbance), 2 (gait disturbance not requiring aid), 3 (inability to walk without using a stick, hand-rail, or walker), and 4 (complete inability to walk). It has been reported that (1) the iNPHGS gait domain score significantly correlates with the timed up-and-go test (TUG) and gait status scale scores, and (2) the gait status scale scores significantly improve in patients whose iNPHGS scores improved after CSF tapping but not in those whose iNPHGS scores did not improve after CSF tapping [15]. Here, we investigated the associations between the preoperative iNPHGS gait domain scores and those of the motor examination section of the UPDRS. We performed the postoperative assessments of parkinsonism in the first week after the VP shunts.

3.2. Evaluation of Radiographic Abnormalities. We performed CT examinations pre- and postoperatively. We investigated the morphologic changes by calculating the values of the Evans index from the CT scans, which are presented in Figure 1(a), the maximum third ventricular width and the midbrain size (anteroposterior and left-toright diameters). The midbrain diameters were measured at the pontomesencephalic junction (Figure 1(d)). The width of the third ventricle was measured at the levels of the superior colliculus and the foramen of Monro (interventricular foramen) (Figures 1(b) and 1(c)). Measurement of these distances on the CT scans was performed with the Xeron PACS system (Xeron healthcare corp., Korea). Each measurement was repeated 3 times, and the average of these triplicate measurements was recorded.

\section{Statistical Analysis}

Statistical analyses were performed with the software, statistical package for social sciences (SPSS) version 17.0 for Windows. Wilcoxon signed rank tests were used to compare pre- and postoperative values of the total scores of the motor examination section in the UPDRS. The effects of VP shunt on the scores for the subitems of the motor examination in the UPDRS, the gait domain of iNPHGS, and the CT findings were analyzed using 2-way analysis of variance with repeated measures followed by Bonferroni post hoc comparisons. Stepwise multiple regression analysis was conducted to determine the association between the 
TABLE 1: Comparison between the grades of the Parkinsonian features before and after ventriculoperitoneal shunt operation.

\begin{tabular}{|c|c|c|c|}
\hline & $\begin{array}{l}\text { Pre VP shunt } \\
(\text { Mean } \pm \mathrm{SD})\end{array}$ & $\begin{array}{c}\text { Post VP shunt } \\
(\text { Mean } \pm \mathrm{SD})\end{array}$ & $P$ value \\
\hline iNPH grading scale gait domain & $2.85 \pm 6.9$ & $1.62 \pm 0.86$ & .001 \\
\hline \multicolumn{4}{|l|}{ UPDRS motor examination subitems } \\
\hline Speech & $0.92 \pm 0.80$ & $0.54 \pm 0.66$ & .200 \\
\hline Facial expression & $1.00 \pm 0.91$ & $0.69 \pm 0.75$ & .305 \\
\hline Tremor at rest & $0.46 \pm 0.78$ & $0.15 \pm 0.38$ & .305 \\
\hline Action tremor of hands & $0.46 \pm 0.66$ & $0.23 \pm 0.44$ & .442 \\
\hline Rigidity & $0.85 \pm 0.80$ & $0.38 \pm 0.65$ & .124 \\
\hline Finger taps & $1.15 \pm 0.90$ & $0.77 \pm 0.73$ & .200 \\
\hline Hand movements & $1.00 \pm 0.82$ & $0.69 \pm 0.75$ & .305 \\
\hline Rapid alternating movements of hands & $1.15 \pm 0.80$ & $0.92 \pm 0.86$ & .442 \\
\hline Leg agility & $0.85 \pm 0.69$ & $0.69 \pm 0.75$ & 608 \\
\hline Arising from chair & $2.69 \pm 1.25$ & $1.23 \pm 0.93$ & .001 \\
\hline Posture & $1.31 \pm 0.48$ & $1.00 \pm 0.00$ & .305 \\
\hline Gait & $2.54 \pm 0.88$ & $1.00 \pm 0.56$ & .001 \\
\hline Postural stability & $2.54 \pm 1.05$ & $1.31 \pm 0.75$ & .001 \\
\hline Body bradykinesia and hypokinesia & $2.15 \pm 0.80$ & $1.46 \pm 0.52$ & .021 \\
\hline UPDRS motor examination total score & $19.08 \pm 5.66$ & $12.00 \pm 7.07$ & $.008^{*}$ \\
\hline
\end{tabular}

VP shunt: ventriculoperitoneal shunt; iNPH: idiopathic normal pressure hydrocephalus; UPDRS: unified Parkinson's disease rating scale; $P$ value: $P$ value by 2 -way analysis of variance with repeated measure, ${ }^{*} P$ value by Wilcoxon signed rank test. The total number of patients was 13.

TABle 2: Item in the motor examination of unified Parkinson's disease rating scale associated with the gait domain of idiopathic normal pressure hydrocephalus grading scale.

\begin{tabular}{lcccc}
\hline & Standardized partial regression coefficient & Partial regression coefficient & $95 \%$ CI & $P$ value \\
\hline Postural stability & 0.815 & 0.535 & $0.3-0.81$ & .001 \\
\hline$P$ val
\end{tabular}

$P$ value by stepwise multiple regression analysis. The total number of patients was 12 .

preoperative scores of the gait domain of the iNPHGS and those of motor examination section of the UPDRS. We also examined the correlations between the preoperative parkinsonism and the radiographic abnormalities by using the canonical correlation analysis. CT finding was treated as a multivariate composite variable represented by the combined influences of the five CT measures shown above and was assessed by examining its relation to a parkinsonism construct as measured by UPDRS. The critical value for statistical significance was set at $P<.05$ for univariate and multivariate analyses.

\section{Results}

Before the VP shunts, the gait domain scores of the iNPHGS were at level 2 in 4 patients, at level 3 in 7 patients, and at level 4 in 2 patients. These scores changed to level 1 in 5 patients and level 2 in 8 patients after the VP shunts. The iNPHGS gait domain scores of all the patients improved after the VP shunts.

Severe impairment was recorded for the subitems of arising from a chair, gait, and postural stability before the VP shunts. In more than $80 \%$ of the patients, the severity of these subitems was graded at higher severity than grade 2. In contrast, the severity of the subitems tremor, rigidity, bradykinesia of extremities, and posture was graded at lower degree of severity than grade 2 .

After the operations, the iNPHGS gait domain scores, the subitems (arising from a chair, gait, postural stability, and body hypokinesia), and the total scores of the motor examination section of the UPDRS improved significantly $(P<.05)$. There were no significant changes in the other subitems of the motor examination section of the UPDRS (Table 1).

The results of stepwise multiple regression analysis to assess the association in the scores between the iNPHGS and UPDRS as the dependent variables are shown in Table 2. We used the following independent variables to avoid the problems caused by multicollinearity: tremor at rest, rigidity, finger taps, hand movements, rapid alternating movements of hands, leg agility, arising from a chair, postural stability, and body hypokinesia. Postural stability in the UPDRS was the only factor that revealed to be a determinant of the gait domain score of the iNPHGS $(P<.05)$.

As seen in Table 3, the postoperative values of Evans indices and the maximum widths of the third ventricle measured at the levels of the superior colliculus were markedly decreased $(P<.05)$, but those measured at the levels of the foramen of Monro and the midbrain diameters did not significantly change as compared to the preoperative values. 


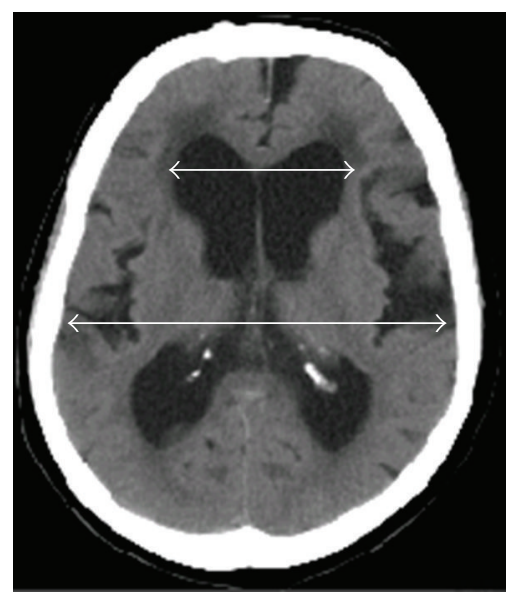

(a)

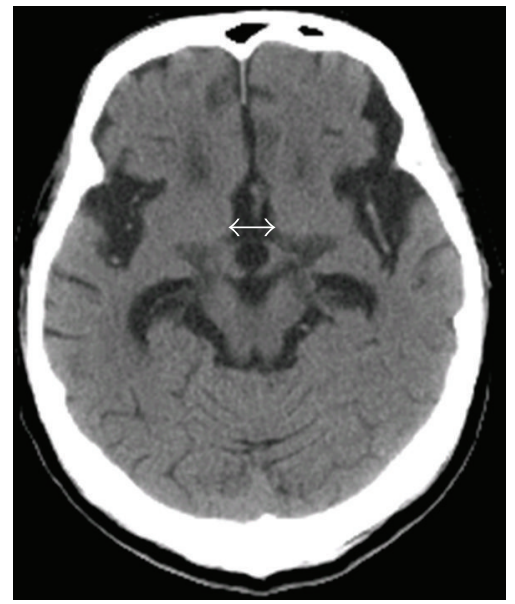

(c)

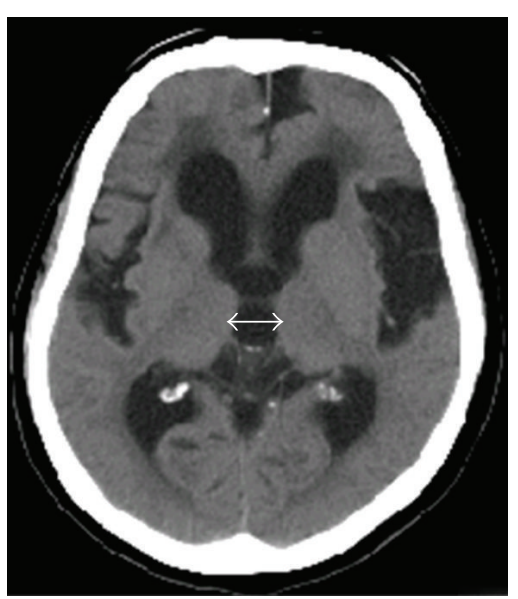

(b)

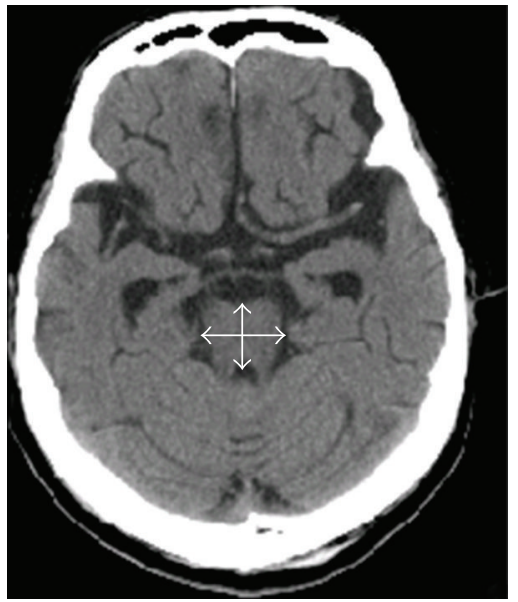

(d)

FIGURE 1: Measurements for iNPH patients on CT scans. (a) Evans index (ratio of maximum width of the frontal horns to the maximum width of the inner table of the cranium); (b) maximum width of the third ventricle at the foramen of Monro (interventricular foramen); (c) maximum width of the third ventricle at the superior colliculus; (d) anteroposterior (vertical) and left-to-right (horizontal) diameters of the midbrain at pontomesencephalic junction.

TABLE 3: Effect of ventriculoperitoneal shunt operation on the computed tomography findings.

\begin{tabular}{|c|c|c|c|}
\hline & $\begin{array}{l}\text { Pre VP shunt } \\
(\text { Mean } \pm \mathrm{SD})\end{array}$ & $\begin{array}{c}\text { Post VP shunt } \\
(\text { Mean } \pm \mathrm{SD})\end{array}$ & $P$ value \\
\hline Evans index & $0.38 \pm 0.047$ & $0.33 \pm 0.43$ & .001 \\
\hline Third ventricle width at superior colliculus & $10.55 \pm 1.80$ & $8.14 \pm 2.03$ & .037 \\
\hline Third ventricle width at foramen of Monro & $12.87 \pm 2.00$ & $11.24 \pm 2.43$ & .155 \\
\hline Anteroposterior diameter of midbrain & $20.86 \pm 2.03$ & $22.47 \pm 1.77$ & .159 \\
\hline Left-to-right diameter of midbrain & $29.64 \pm 2.82$ & $29.59 \pm 2.14$ & .967 \\
\hline
\end{tabular}

$P$ value by 2-way analysis of variance with repeated measure. The total number of patients was 12 .

In canonical correlation analysis, the correlation between the following independent variables was used to reduce the impact of multicollinearity: the subitem scores (arising from a chair, postural stability, and body hypokinesia) and total scores of the motor examination section in the UPDRS. In the first canonical variates of canonical correlation analysis (canonical correlation coefficient $=0.996, P<.045$ ), the Evans index had a large influence on the postural stability (Table 4).

\section{Discussion}

6.1. Characteristics of Parkinsonism in iNPH. The clinical guidelines for iNPH generally recommend the timed upand-go (TUG) test for the assessment of disease severity [4]. However, some iNPH patients with severe postural instability are unable to perform the TUG test. In our study, several items of the UPDRS motor examination section proved to be useful in the evaluation of the clinical severity of 
TABLE 4: Canonical correlations between the grades of the Parkinsonian features responding to the ventriculoperitoneal shunt operation and computed tomography findings.

\begin{tabular}{ccc}
\hline & First canonical variate \\
\hline CT finding set & Correlation & \\
Third ventricle width at superior colliculus & -0.23 & 0.908 \\
Third ventricle width at foramen of Monro & -0.612 & -1.011 \\
Anteroposterior diameter of midbrain & 0.094 \\
Left-to-right diameter of midbrain & -0.162 \\
Evans index & -0.814 \\
Percent of variance & 0.225 \\
Redundancy index & 0.223 & -0.504 \\
Parkinsonism set (assessed by UPDRS) & & -0.564 \\
Total score of motor examination & -0.59 & -1.151 \\
Subitem score of postural stability & -0.592 \\
arising from chair & -0.499 \\
body bradykinesia and hypokinesia & 0.356 \\
Percent of variance & 0.27 \\
Redundancy index & 0.266 \\
Canonical Correlation & 0.996 \\
\hline
\end{tabular}

CT: computed tomography; $n$ : number of subjects; UPDRS: unified Parkinson's disease rating scale; Percent of variance $=$ average squared loading $\left(L^{2}\right)$; Redundancy index = average $L^{2}$. canonical $R^{2}$. The total number of patients was 11 .

the characteristic parkinsonism in iNPH, and the patients showed actual improvements in those items after undergoing the VP shunts (Table 1). These shunt-responsive items can reflect the characteristics of the parkinsonism.

The gait domain scores of the iNPHGS were significantly correlated with the scores of postural stability in the motor examination section of the UPDRS $(P<.001)$ (Table 2$)$. The high association between the improvement in the postural stability score and in the Evans index after the VP shunt and our results indicate that postural stability, a subitem of the UPDRS motor examination section, might be a specific feature closely related to the gait disturbance in iNPH and that the postural instability may be a cardinal feature of iNPH gait.

Knutsson and Lying-Tunell suggested that the gait disturbances in NPH are similar to gait apraxia [16]. Bugalho and Guimaraes reported that the response of postural instability to CSF tap test was weaker than that of hypokinesia and did not correlate with that of step length or gait velocity in iNPH [17]. However, consistent with the findings from other studies, our results indicate that the gait disturbance in iNPH may be predominantly associated with postural instability rather than hypokinesia $[5,18]$. Therefore, we speculate that the short-steppage gait in iNPH may be associated with postural instability.

6.2. Improvement in Radiographic Findings. Mocco et al. reported significant increases in the anteroposterior and the left-to-right midbrain diameters but no significant change in the size of the ventricles after shunt operation, stating that gait disturbance in iNPH may be strongly associated with midbrain atrophy [19]. However, in our patients with definite iNPH, the dilatation of the lateral and third ventricles at the levels of the superior colliculus was significantly improved, but the midbrain diameters did not change significantly after a VP shunt.

Our results indicate that CSF loss improved the dilatation of the lateral and third ventricles at the level of the superior colliculus and that these morphologic alterations and not the decreased midbrain diameters are the characteristic features in iNPH.

6.3. Correlation between Parkinsonism and Radiographic Abnormalities. Lee et al. reported that the reduction in the maximal anteroposterior diameter of the midbrain was significantly correlated with the severity of gait disturbance, and no significant correlation was observed between gait disturbance and the changes in the diameter of the lateral or third ventricle [9]. However, the results of canonical correlation analysis showed that the postural instability was a cardinal feature of gait disturbance in iNPH, and it was influenced by ventricle widths rather than midbrain diameters. They also suggested that the gait disturbances may be associated with postural instability in iNPH as well as in progressive supranuclear palsy (PSP) or advanced Parkinson's disease (PD) [20] and that the neuronal dysfunction in pedunculopontine nucleus (PPN), the major component of the mesencephalic locomotor region, may be important in the pathophysiology of locomotor and postural disturbances. Although loss of neurons in the PPN was reported in PSP and advanced PD [20], there are no reports of the PPN pathology in iNPH to our knowledge. Our results indicate that the lesions involved in the gait disturbance in the case of iNPH patients are not specific to the midbrain (Table 4).

Our results indicate that Evans index had a large influence on the postural stability (Table 4 ). In addition, 
patients with iNPH are known to have frequent frontal release signs, and an association has been reported between frontal cognitive impairment and gait disturbance [21]. In this context, an association may exist between frontal lobe dysfunction and the postural instability in iNPH. It is possible that the postural instability in iNPH is associated with impaired frontal projections descending close to the frontal horns of the lateral ventricles toward the reticular formation in the tegmentum of the brainstem [22].

Cerebral blood flow (CBF) studies in iNPH have shown a decrease in the BG blood flow, and this decrease has been found to be correlated with gait disturbance [23, 24]. Pathological changes in the BG have been reported in postmortem brains of the iNPH patients [25]. However, it remains controversial whether the mechanism of the gait disturbance can be explained only by the functional defect in the BG loop. Stolze et al. stated that the poor response to external cues for improving gait velocity and step length indicate that the BG-supplementary motor area (SMA) loop may not be a major lesion causing the gait disturbance in iNPH [5].

Our study has several limitations. First, we used a small sample size. Second, the retrospective design of our study may introduce a selection bias. Third, since the assessments of parkinsonism were semiquantitative, the accuracy of assessments may be poor. Further study using kinetic analysis with more iNPH patients is needed to investigate the gait disturbance or postural instability. Finally, although CT remains the standard examination for postoperative imaging in a clinical setting to investigate the brain morphological alterations in iNPH, the image resolution obtained in CT is markedly lower than that in MRI.

\section{Acknowledgments}

The authors thank the iNPH patients for participating in this research. This research was supported in part by grant-in-aid from the Ministry of Education, Culture, Sports Science and Technology of Japan (20591024).

\section{References}

[1] S. Hakim and R. D. Adams, "The special clinical problem of symptomatic hydrocephalus with normal cerebrospinal fluid pressure. Observations on cerebrospinal fluid hydrodynamics," Journal of the Neurological Sciences, vol. 2, pp. 307-327, 1965.

[2] L. Gunasekera and A. E. Richardson, "Computerized axial tomography in idiopathic hydrocephalus," Brain, vol. 100, pp. 749-754, 1977.

[3] K. Mori, "Management of idiopathic normal-pressure hydrocephalus: a multiinstitutional study conducted in Japan," Journal of Neurosurgery, vol. 95, pp. 970-973, 2001.

[4] M. Ishikawa, "Clinical guidelines for idiopathic normal pressure hydrocephalus," Neurologia Medico-Chirurgica, vol. 44, pp. 222-223, 2004.

[5] H. Stolze, J. P. Kuhtz-Buschbeck, H. Drucke, K. Johnk, M. Illert, and G. Deuschl, "Comparative analysis of the gait disorder of normal pressure hydrocephalus and parkinson's disease," Journal of Neurology, Neurosurgery and Psychiatry, vol. 70, no. 3, pp. 289-297, 2001.

[6] P. D. Thompson and J. G. Nutt, "Frontal and higher level gait disorders," in Clinical Disorders of Balance, Posture and Gait, A. M. Bronstein, T. Brandt, M. H. Woollacott, and J. G. Nutt, Eds., pp. 216-222, Arnold, London, UK, 2nd edition, 2004.

[7] H. Kitagaki, E. Mori, K. Ishii, S. Yamaji, N. Hirono, and T. Imamura, "CSF spaces in idiopathic normal pressure hydrocephalus: morphology and volumetry," American Journal of Neuroradiology, vol. 19, pp. 1277-1284, 1998.

[8] M. Tullberg, C. Jensen, S. Ekholm, and C. Wikkelso, "Normal pressure hydrocephalus: vascular white matter changes on MR images must not exclude patients from shunt surgery," American Journal of Neuroradiology, vol. 22, pp. 1665-1672, 2001.

[9] P. H. Lee, S. W. Yong, Y. H. Ahn, and K. Huh, "Correlation of midbrain diameter and gait disturbance in patients with idiopathic normal pressure hydrocephalus," Journal of Neurology, vol. 252, pp. 958-963, 2005.

[10] P. Bugalho and L. Alves, "Normal-pressure hydrocephalus: white matter lesions correlate negatively with gait improvement after lumbar puncture," Clinical Neurology and Neurosurgery, vol. 109, pp. 774-778, 2007.

[11] T. Curran and A. E. Lang, "Parkinsonian syndromes associated with hydrocephalus: case reports, a review of the literature, and pathophysiological hypotheses," Movement Disorders, vol. 9, pp. 508-520, 1994.

[12] B. A. Racette, G. J. Esper, J. Antenor, et al., "Pathophysiology of parkinsonism due to hydrocephalus," Journal of Neurology, Neurosurgery and Psychiatry, vol. 75, no. 11, pp. 1617-1619, 2004.

[13] A. S. Mandir, J. Hilfiker, G. Thomas, et al., "Extrapyramidal signs in normal pressure hydrocephalus: an objective assessment," Cerebrospinal Fluid Research, vol. 4, no. 11, pp. 16171619, 2007.

[14] S. Fahn and R. Elton, "Unified Parkinson's disease rating scale," in Recent Developments in Parkinson's Disease, S. Fahn, C. D. Marsden, and M. Goldstein, Eds., vol. 2, Macmillian Healthcare Information, Florham Park, NJ, USA, 1987.

[15] Y. Kubo, H. Kazui, T. Yoshida, et al., "Validation of grading scale for evaluating symptoms of idiopathic normal-pressure hydrocephalus," Dementia and Geriatric Cognitive Disorders, vol. 25, no. 1, pp. 37-45, 2008.

[16] E. Knutsson and U. Lying-Tunell, "Gait apraxia in normalpressure hydrocephalus: patterns of movement and muscle activation," Neurology, vol. 35, pp. 155-160, 1985.

[17] P. Bugalho and J. Guimaraes, "Gait disturbance in normal pressure hydrocephalus: a clinical study," Parkinsonism and Related Disorders, vol. 13, pp. 434-437, 2006.

[18] E. Blomsterwall, U. Svantesson, U. Carlsson, M. Tullberg, and C. Wikkelso, "Postural disturbance in patients with normal pressure hydrocephalus," Acta Neurologica Scandinavica, vol. 102, pp. 284-291, 2000.

[19] J. Mocco, M. I. Tomey, R. J. Komotar, et al., "Ventriculoperitoneal shunting of idiopathic normal pressure hydrocephalus increases midbrain size: a potential mechanism for gait improvement," Neurosurgery, vol. 59, no. 4, pp. 847-850, 2006.

[20] P. A. Pahapill and A. M. Lozano, "The pedunculopontine nucleus and Parkinson's disease," Brain, vol. 123, no. 9, pp. 1763-1783, 2000.

[21] N. Miyoshi, H. Kazui, A. Ogino, et al., "Association between cognitive impairment and gait disturbance in patients with idiopathic normal pressure hydrocephalus," Dementia and Geriatric Cognitive Disorders, vol. 20, pp. 71-76, 2005. 
[22] W. G. Bradley, "Normal pressure hydrocephalus: new concepts on etiology and diagnosis," American Journal of Neuroradiology, vol. 21, no. 9, pp. 1586-1590, 2000.

[23] B. K. Owler, S. Momjian, Z. Czosnyka, et al., "Normal pressure hydrocephalus and cerebral blood flow: a PET study of baseline values," Journal of Cerebral Blood Flow and Metabolism, vol. 24, no. 1, pp. 17-23, 2004.

[24] Y. Ouchi, T. Nakayama, T. Kanno, E. Yoshikawa, T. Shinke, and T. Torizuka, "In vivo presynaptic and postsynaptic striatal dopamine functions in idiopathic normal pressure hydrocephalus," Journal of Cerebral Blood Flow and Metabolism, vol. 27, no. 4, pp. 803-810, 2007.

[25] K. Akai, S. Uchigasaki, U. Tanaka, and A. Komatsu, "Normal pressure hydrocephalus. Neuropathological study," Acta Pathologica Japonica, vol. 37, no. 1, pp. 97-110, 1987. 


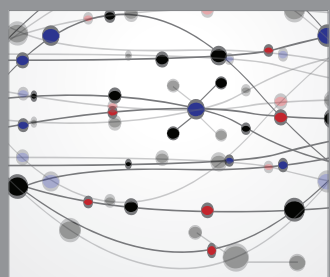

The Scientific World Journal
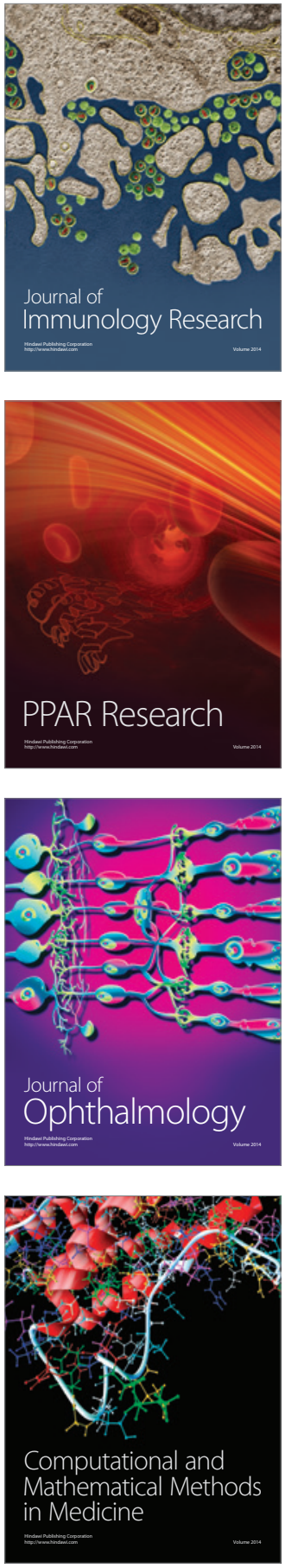

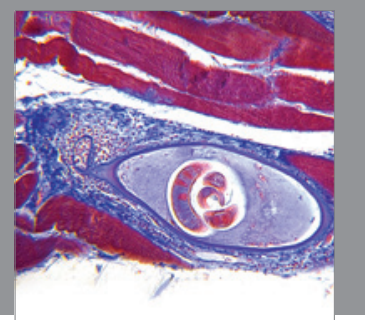

Gastroenterology

Research and Practice
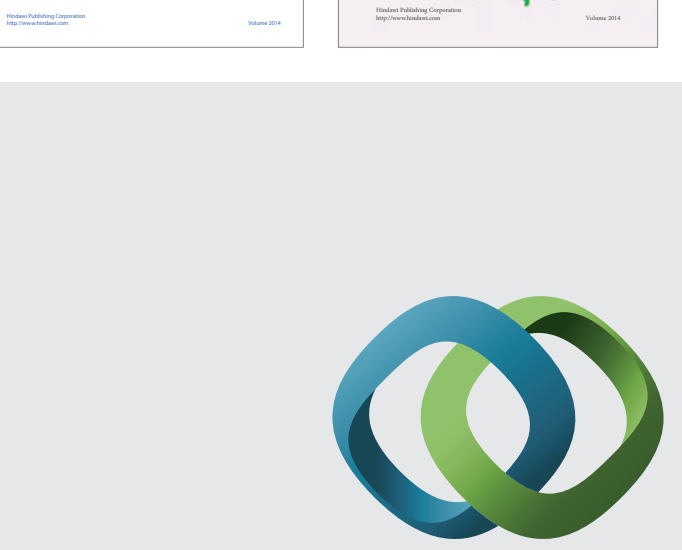

\section{Hindawi}

Submit your manuscripts at

http://www.hindawi.com
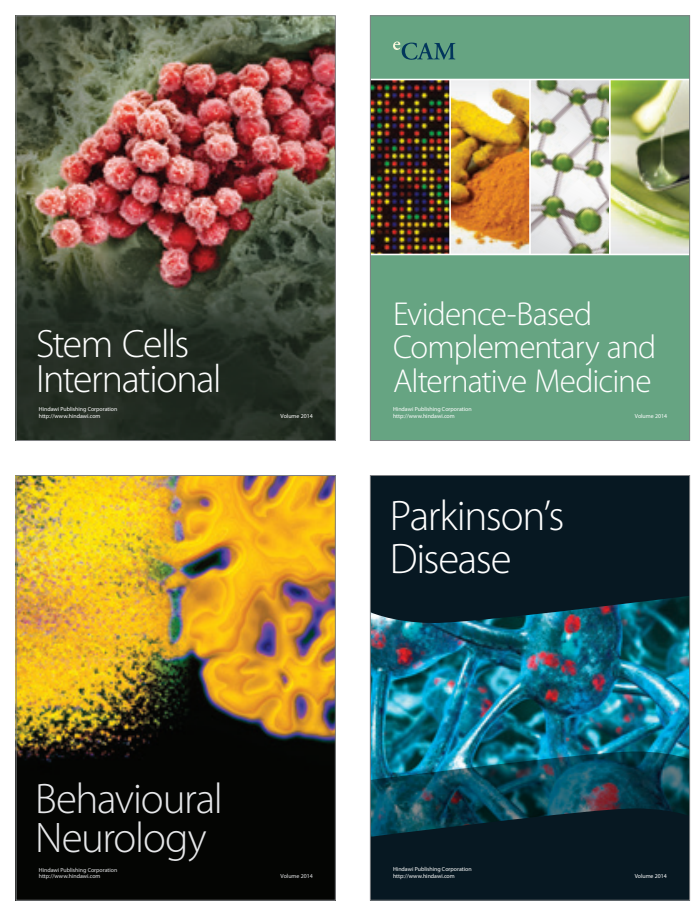

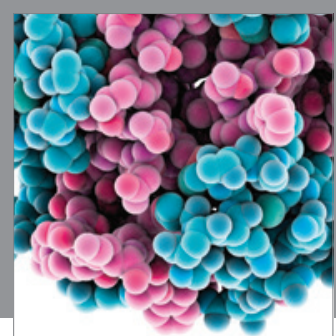

Journal of
Diabetes Research

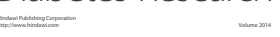

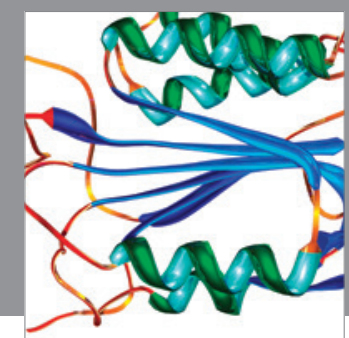

Disease Markers
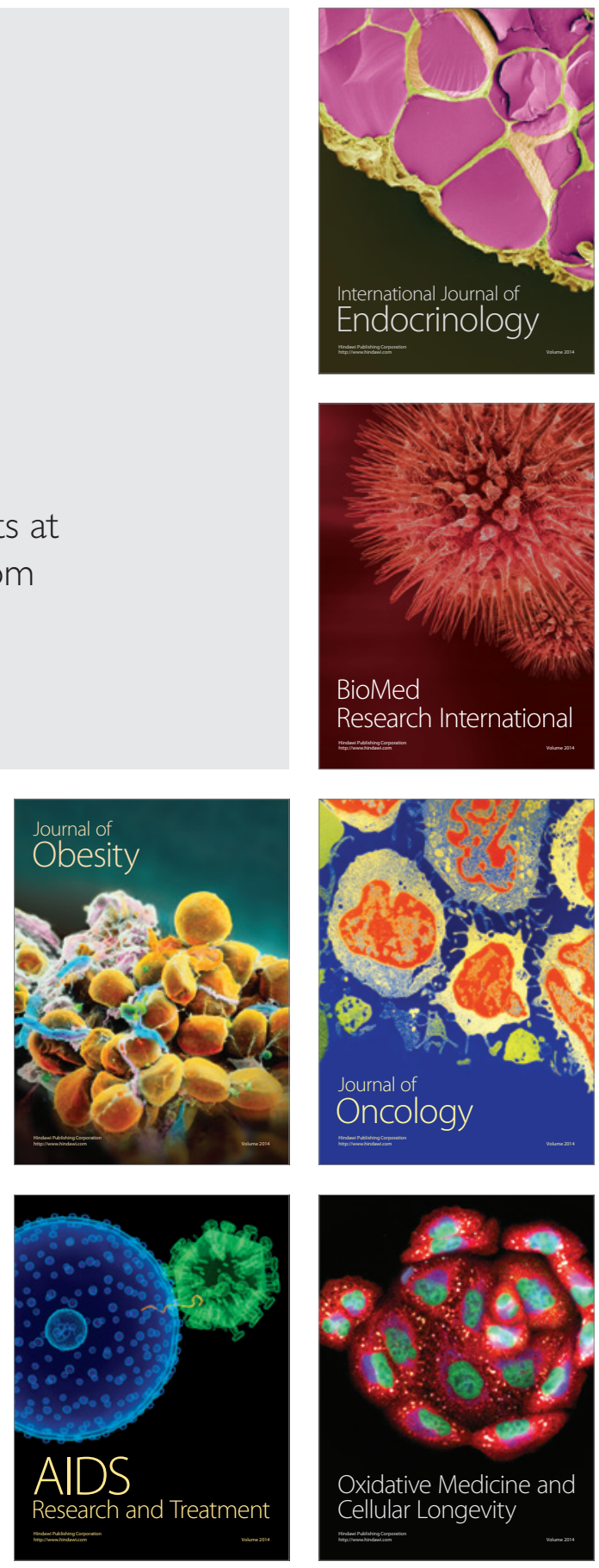